\title{
Cotransfected human chondrocytes: over-expression of IGF-I and SOX9 enhances the synthesis of cartilage matrix components collagen-II and glycosaminoglycans
}

\author{
M. Simental-Mendía ${ }^{1}$, J. Lara-Arias ${ }^{2}$, E. Álvarez-Lozano ${ }^{2}$, S. Said-Fernández ${ }^{1}$, \\ A. Soto-Domínguez ${ }^{3}$, G.R. Padilla-Rivas ${ }^{1}$ and H.G. Martínez-Rodríguez ${ }^{1}$ \\ ${ }^{1}$ Department of Biochemistry and Molecular Medicine, Faculty of Medicine \\ Autonomous University of Nuevo León, Monterrey, NL, Mexico \\ ${ }^{2}$ Laboratory of Tissue Engineering, Bone and Tissue Bank, Universitary Hospital, \\ Autonomous University of Nuevo León, Monterrey, NL, Mexico \\ ${ }^{3}$ Department of Histology, Faculty of Medicine, Autonomous University of Nuevo León, Monterrey, NL, Mexico
}

\begin{abstract}
Damage to cartilage causes a loss of type II collagen (Col-II) and glycosaminoglycans (GAG). To restore the original cartilage architecture, cell factors that stimulate Col-II and GAG production are needed. Insulin-like growth factor I (IGF-I) and transcription factor SOX9 are essential for the synthesis of cartilage matrix, chondrocyte proliferation, and phenotype maintenance. We evaluated the combined effect of IGF-I and SOX9 transgene expression on Col-II and GAG production by cultured human articular chondrocytes. Transient transfection and cotransfection were performed using two mammalian expression plasmids (pCMV-SPORT6), one for each transgene. At day 9 post-transfection, the chondrocytes that were overexpressing IGF-I/SOX9 showed 2-fold increased mRNA expression of the Col-II gene, as well as a $57 \%$ increase in Col-II protein, whereas type I collagen expression ( $\mathrm{Col}-\mathrm{I})$ was decreased by $59.3 \%$ compared with controls. The production of GAG by these cells increased significantly compared with the controls at day 9 (3.3- vs 1.8-times, an increase of almost $83 \%$ ). Thus, IGF-I/SOX9 cotransfected chondrocytes may be useful for cell-based articular cartilage therapies.
\end{abstract}

Key words: Type II collagen; Glycosaminoglycans; IGF-I/SOX9 transgenes; Human chondrocytes; Articular cartilage; Cotransfection

\section{Introduction}

Articular cartilage is an avascular and highly organized tissue characterized by a low cell density, complex biomechanical properties, and a poor capacity for healing. After injury, type II collagen (Col-II) and glycosaminoglycans (GAG), two major and essential components of the cartilage extracellular matrix, are lost. The repair tissue that forms is not hyaline cartilage, but instead a fibrocartilage rich in type I collagen (Col-I) (1), which eventually fails (2). Therefore, a method that can induce proper repair of damaged cartilage is needed. The current surgical interventions, including microfracture, chondral grafts, and chondrocyte transplantation, among others, are unable to restore the original cartilage surface (3). To re-establish the structural integrity of hyaline cartilage after injury, the transfer of genes encoding factors that increase cell proliferation and their differentiation into articular chondrocytes has been proposed (4). The regeneration of articular cartilage is a complex process that requires stimulation by several chondrogenic factors. Thus, a therapeutic strategy based on the delivery of multiple recombinant genes may induce better functional repair (5). Because of their chondro-regenerative effects, genes coding for fibroblast growth factor 2 (FGF-2) (6), transforming growth factor- $\beta$ (TGF- $\beta$ ) (7), bone morphogenetic proteins 7 and 2 (BMP-7 and BMP-2) (8), insulin-like growth factor I (IGF-I) (9), and the transcription factor SOX9 (10) have been transferred to chondrocytes, both individually and in combination $(5,11)$. Encouraging results have been reported with the use of combined, rather than single, gene transfer $(4,12)$. However, the simultaneous

Correspondence: H.G. Martínez-Rodríguez: < herminia.martinezrd@uanl.edu.mx>; <herminiamar@gmail.com>. 
effects of IGF-I and SOX9, two of the major factors involved in the matrix synthesis by chondrocytes in human articular cartilage, have not been reported. IGF-I induces specific anabolic effects on cartilage explants and chondrocyte monolayers. Chondrocytes transfected with the IGF-I gene exhibit an increased synthesis of large proteoglycan aggregates that are partially composed of GAG and Col-II $(7,9)$. In addition, SOX9 is a transcription factor capable of shifting the metabolic balance towards the synthesis of hyaline cartilage matrix components, as well as stimulating chondrocyte differentiation (12).

Most gene transfection for stimulating cartilage repair is done using viruses $(12,13)$. These vectors have long-term expression, but can produce effects with undesirable consequences, such as the induction of systemic inflammatory response syndrome after systemic administration of adenoviral vectors and deregulation of T-cell proliferation driven by retrovirus enhancer activity (14). Transient cotransfection using plasmids as vectors has produced promising results (15). The plasmids remain active inside the cells for only a short, but sufficient, amount of time to express the desired genes, leading to subsequent cartilage healing (16) while reducing the potential risks to patients.

In the present study, we performed a transient cotransfection of chondrocytes with plasmids carrying the cDNA for IGF-I and SOX9 to create cotransfected chondrocytes (CTC). We quantified the expression of IGF-I and SOX9 and evaluated the effect of those two factors on Col-II and GAG synthesis in the CTC compared with those of non-transfected chondrocytes (NTC) and chondrocytes transfected with only one plasmid coding for IGF-I (IGF-I-TC) or SOX9 (SOX9-TC). We also determined the effects of IGF-I, SOX9, or both factors on the synthesis of Col-I, which is a major component of the bone matrix, but not of hyaline cartilage (17).

\section{Material and Methods}

\section{IGF-I and SOX9 plasmid vectors}

A pCMV-SPORT6 plasmid backbone (Open Biosystems Inc., USA) was used to construct the pCMVSPORT6 IGF-I and pCMV-SPORT6 SOX9 plasmids. IGF-I cDNA was cloned into the mammalian pCMVSPORT6 plasmid using the restriction enzymes Notl and Sall (Invitrogen, USA). The same construction process was followed to create a pCMV-SPORT6 EGFP plasmid carrying cDNA for enhanced green fluorescent protein (EGFP). The EGFP sequence was obtained from the pIRES2-EGFP plasmid (kindly provided by Dr. Martín Canizales, MD, Anderson Cancer Center, Houston, TX, USA). The vector pCMV-SPORT6 contained the selectable ampicillin-resistant gene and pUC origin, which allowed for plasmid amplification in Escherichia coli TOP 10. The pCMV-SPORT6 SOX9, pCMV-SPORT6 IGF-I, and pCMV-SPORT6 EGFP plasmids were purified through a silica membrane column with a Plasmid Midi Kit (Qiagen, USA).

\section{Human chondrocyte isolation and culture}

Human chondrocytes were obtained from the unused portions of three cartilage biopsies. The cells were recovered using successive cartilage digestions with $0.25 \%$ trypsin and $2 \mathrm{mg}$ of type II collagenase $/ \mathrm{mL}$ (Sigma-Aldrich Co., USA). The chondrocytes were suspended in opti-MEM medium supplemented with $10 \%$ fetal bovine serum, gentamicin $(0.05 \mathrm{mg} / \mathrm{mL})$, and amphotericin B (50 ng/mL; complete medium), all purchased from Gibco $^{\circledR}$ (Thermo Fisher Scientific, USA). When the monolayers reached $80 \%$ confluence, the chondrocytes were harvested, washed three times with phosphate buffered saline (Sigma-Aldrich Co.), and their concentration was adjusted to $2 \times 10^{5}$ cells $/ \mathrm{mL}$ in complete medium. Aliquots of the cell suspension were seeded into wells of a 6-well culture plate (Corning Incorporated, USA) containing $1 \mathrm{~mL}$ of complete medium and incubated at $37^{\circ} \mathrm{C}$ for $24 \mathrm{~h}$ in a $5 \% \mathrm{CO}_{2}$ atmosphere.

\section{Chondrocyte transfection}

The chondrocyte monolayers at $80 \%$ confluence were transfected with a mixture of the plasmid pCMV-SPORT6 EGFP and the FuGENE 6 transfection reagent (Roche Applied Bioscience, USA) using 1:3, 2:3, and 1:6 ratios of plasmid $\mu \mathrm{g}$ to FuGENE $\mu \mathrm{L}$ and incubated at $37^{\circ} \mathrm{C}$ in a $5 \% \mathrm{CO}_{2}$ atmosphere for $48 \mathrm{~h}$. The number of fluorescent chondrocytes was determined using a Nikon $50 \mathrm{i}$ microscope with an epifluorescence illuminator (Nikon Instruments Inc., USA) at a magnification of $20 \times$. The transfection efficiency was reported as the percentage of fluorescent cells calculated with respect to the total number of cells observed in eight randomly selected microscopic fields. This experiment was performed in triplicate.

Chondrocyte transfection with pCMV-SPORT6 SOX9 and PCMV-SPORT6 IGF-I was performed using $1.0 \mu \mathrm{g}$ of plasmid DNA and $3.0 \mu \mathrm{L}$ of FuGENE 6 reagent. The cotransfection with both plasmids was performed using $2.0 \mu \mathrm{g}$ of plasmid DNA (1.0 $\mu \mathrm{g}$ pCMV-SPORT6 SOX9 and $1.0 \mu \mathrm{g}$ pCMV-SPORT6 IGF-I) and $6.0 \mu \mathrm{L}$ of FuGENE6 transfection reagent per well. These preparations were incubated for 3,6 , or 9 days at $37^{\circ} \mathrm{C}$ in a $5 \% \mathrm{CO}_{2}$ atmosphere. All chondrocyte transfections were performed according to the instructions provided by the FUGENE 6 transfection reagent manufacturer.

\section{Stimulation of proliferation by IGF-I and SOX 9}

We counted the numbers of NTC, IGF-I-TC, SOX9-TC, and CTC with a Nikon microscope (Nikon Instruments) at a magnification of $40 \times$ in eight randomly selected fields per microplate well.

\section{Total RNA extraction and RT-PCR}

On days 3, 6, and 9 post-transfection (PT), the total RNA was isolated from transfected, CTC and NTC using an RNA cell and tissue purification kit (GENTRA Systems, 
Table 1. Primers used for the amplification of the GAPDH, IGF-I, and SOX9 sequences.

\begin{tabular}{llc}
\hline Oligonucleotide & \multicolumn{1}{c}{ Sequence } & Amplicon length (bp) \\
\hline GAPDH & Fw: 5'-AAGATGGCCCAGGAGAACCCCAAG-3' & 980 \\
& Rv: 5'-TAATCCTTCATGTGCACCGCCCTG-3' & \\
IGF-I & Fw: 5'-CAGAATTCACAATGGGAAAAATCAGCAGTCTTCC-3' & 473 \\
\multirow{2}{*}{ SOX9 } & Rv: 5'-CTAGATGTCGACATCCTGTAGTTCTTGTTTCCTG-3' & \multirow{2}{*}{835} \\
& Fw: 5'-TCATGAAGATGACCGACGAGCA-3' & \\
& Rv: 5'-TTGGAGATGACGTCGCTGCTCA-3' & \\
\hline
\end{tabular}

GAPDH: glyceraldehyde-3-phosphate dehydrogenase; Fw: forward primer; Rv: reverse primer.

USA). cDNA was synthesized from each RNA preparation using M-MLV reverse transcriptase (Invitrogen), and the total RNA (500 ng) was treated with $0.5 \mathrm{U}$ of deoxyribonuclease I (DNase I; Invitrogen) to digest genomic DNA. The genes SOX9, IGF-I, and the constitutive gene glyceraldehyde-3-phosphate dehydrogenase (GAPDH) were amplified by polymerase chain reaction (PCR) using specific primers (Table 1).

The amplification products were separated by electrophoresis in a $1.5 \%$ agarose gel (Invitrogen) and stained with $5 \mu \mathrm{L}$ of ethidium bromide at a concentration of $0.5 \mathrm{mg} / \mathrm{mL}$ (Sigma-Aldrich Co.). The PCR products were visualized on a gel documentation system (UVP, Model M-26E; USA). A densitometric analysis was performed with the Phoretix 1D software (TotalLab Ltd., UK; available online: < http://www.totallab.com/1d-downloads/>). The density of each band was expressed as a value normalized to the average GAPDH cDNA band density in each gel. The density of the cDNA bands were expressed as their total number of pixels, and the level of mRNA expression was assumed to be equivalent to the density of the respective bands.

\section{Quantitative RT-PCR}

The total RNA and cDNA were prepared according to the methods described above. The reactions were performed on a 7500 fast real-time PCR system using MicroAmp 96-well reaction plates and TaqMan Universal PCR Master Mix. Specific TaqMan probes were used to detect the expression of Col-I (Hs00264051_m1) and Col-Il (Hs00264051_m1) with GAPDH as the internal control (Hs02758991_g1). A gene expression analysis was performed using the comparative $\mathrm{C}_{\mathrm{T}}$ method $\left(\Delta \Delta \mathrm{C}_{\mathrm{T}}\right)$. All instruments, laboratory materials, and chemicals used during the qRT-PCR experiments were purchased from Applied Biosystems (USA).

\section{Immunolabeling for Col-I and Col-II}

The suspensions of CTC, SOX9-TC, IGF-I-TC, and NTC were adjusted to densities of $2 \times 10^{4}$ cells $/ \mathrm{mL}$ in complete medium, and $500 \mu \mathrm{L}$ aliquots were seeded into each compartment of a four-well microchamber slide (NuncTM; Thermo Fisher Scientific). One microchamber slide was prepared for each transfection condition, and the microchamber slides were immediately incubated at $37^{\circ} \mathrm{C}$ in a $5 \% \mathrm{CO}_{2}$ atmosphere for 3,6 , or 9 days. Next, the culture medium was removed and the cells were fixed with methanol-acetone $(1: 1 \mathrm{v} / \mathrm{v})$ for $20 \mathrm{~min}$ at $-20^{\circ} \mathrm{C}$. The NTC, IGF-I-TC, SOX9-TC, and CTC on the 3rd, 6th, and 9th PT days were incubated with monoclonal antibodies against both Col-I (ab23446; Abcam, Inc., USA) and Col-II (ab34712; Abcam, Inc.), and positive staining was detected using mouse- and rabbit-specific HRP/DAB detection IHC Kit (Abcam, Inc.), according to the manufacturer's instructions.

\section{Immunolabeling analysis}

The immunocytochemistry preparations were examined at $40 \times$ with a Nikon microscope (Nikon Instruments) equipped with a digital camera (Labpohot 2; Nikon Instruments) with a resolution of $1600 \times 1200$ pixels. Eight fields in each well of the chamber were randomly chosen and imaged. The color photomicrographs were stored in the NIS-elements BR 2.30 software (Nikon Instruments) and digitally binarized. The background was uniformly eliminated with a digital filter and the cell staining intensities were analyzed with ImageJ software (National Institutes of Health, USA). The intensity of the immunolabeling is reported as the means $\pm S D$ of the total pixels normalized to the number of cells in the measurement area $(n=32$ in each chamber slide).

\section{GAG analysis}

The accumulated free GAG in the chondrocyte-conditioned culture media from all culture conditions was measured following the dimethyl-methylene blue spectrophotometric assay using the Rheumera GAG detection kit (Astarte Biologics, USA), according to the manufacturer's instructions. Chondroitin sulfate provided with the kit was used as the standard. The total protein concentration was determined by the Bradford method in each assayed culture medium and used to normalize the GAG quantifications.

\section{Ethics}

Written informed consent was obtained from all patients even though this study posed no risk to the 
patients because it was performed using surplus material donated by patients who were undergoing knee chondrografts. The protocol for this study was approved by the Research and Ethical Committee of the Medicine Faculty of the Universidad Autónoma de Nuevo León (registry number: OR03-053).

\section{Statistical analysis}

All results are reported as mean $\pm S D$ of three independent experiments performed in triplicate $(n=9)$. Two-way ANOVA with Bonferroni's post hoc tests were performed for all experiments except for the stimulation of proliferation experiment, which was assessed using Student's $t$-tests. Statistical analyses were performed in GraphPad Prism version 5.00 for Windows (GraphPad Software, Inc., USA). P-values less than 0.05 were considered to be statistically significant.

\section{Results}

\section{Transfection efficiency}

The efficiency of chondrocyte transfection with pCMVSPORT6 EGFP was $65.8 \pm 10.65$ at $3: 1,53.2 \pm 4.43$ at $3: 2$, and $52.5 \pm 5.13 \%$ at $6: 1$ ratios of FuGENE 6 transfection reagent to pCMV-SPORT6 EGFP. Although no significant differences in transfection efficiency with ratio were found, the $3: 1$ ratio was chosen for all subsequent transfection experiments.

\section{Effects of IGF-I and SOX9 on proliferation}

On day 3 PT, the NTC, IGF-I-TC, SOX9-TC, and CTC showed similar numbers of cells per microscope field $(19.9 \pm 4.2,20.8 \pm 4.4,20.1 \pm 4.9$, and 33.0 \pm 7.6$)$. However, on day $9 \mathrm{PT}$, the IGF-I-TC cultures contained 47.2 more $(153.2 \pm 25.6$ cells) and the CTC cultures contained 54.8 more $(161.1 \pm 42.2$ cells) cells than the NTC
(104.1 \pm 19.7 cells) cultures. These increases in the number of cells were both statistically significant $(P<0.05)$.

\section{Expression of IGF-I, SOX9, and GAPDH}

As shown in Figure 1A, the expression of IGF-I by the NTC and SOX9-TC was not significantly changed from days 3 to $9 \mathrm{PT}$. In contrast, from the 3rd day until the 9th day PT, significantly higher IGF-I expression was found by IGF-I-TC (4.2-fold) and CTC (5.5-fold) compared with that of the NTC or SOX9-TC. No significant differences in IGF-I expression were observed between the IGF-I-TC and the CTC or between the SOX9-TC and NTC. The expression of SOX9 was noticeably lower by SOX9-TC (2.7-4.1-fold) and CTC (1.5-2.0-fold) compared with their expression of IGF-I. The expression of IGF-I by CTC on day 9 PT was 3.7-times higher than that by NTC. On days 6 and 9 PT, all cultures maintained a steady level of SOX9 expression. In addition, throughout the observation period, the expression of SOX9 was significantly higher by the SOX9-TC (3.6-4.2-fold) and CTC (3.5-3.75-fold) than that of the NTC (3.5-3.75-fold), as well as that by the IGF-I-TC (2.5-3.7-fold; Figure 1B). The expression of SOX9 by the CTC on day 9 PT was 2.7 times higher than that by the NTC. The expression of GAPDH was not noticeably changed with any culture condition.

\section{RT-PCR quantitation of $\mathrm{Col}-\mathrm{I}$ and $\mathrm{Col}-\mathrm{Il}$ expression}

As shown in Figure 2A, Col-l expression appeared to decrease by $16.7 \%$ (SOX9-TC) and $31.4 \%$ (CTC) compared with the NTC expression on day 6 PT, but these differences were not statistically significant. However, the expression of Col-I was only significantly decreased in the CTC compared with that expressed by the NTC (59.3\%) at day 9 PT. In contrast, the expression of Col-ll (Figure 2B) showed a clear and consistent increase from days 3 to 9 PT in all cultures. The increased expressions of Col-Il by
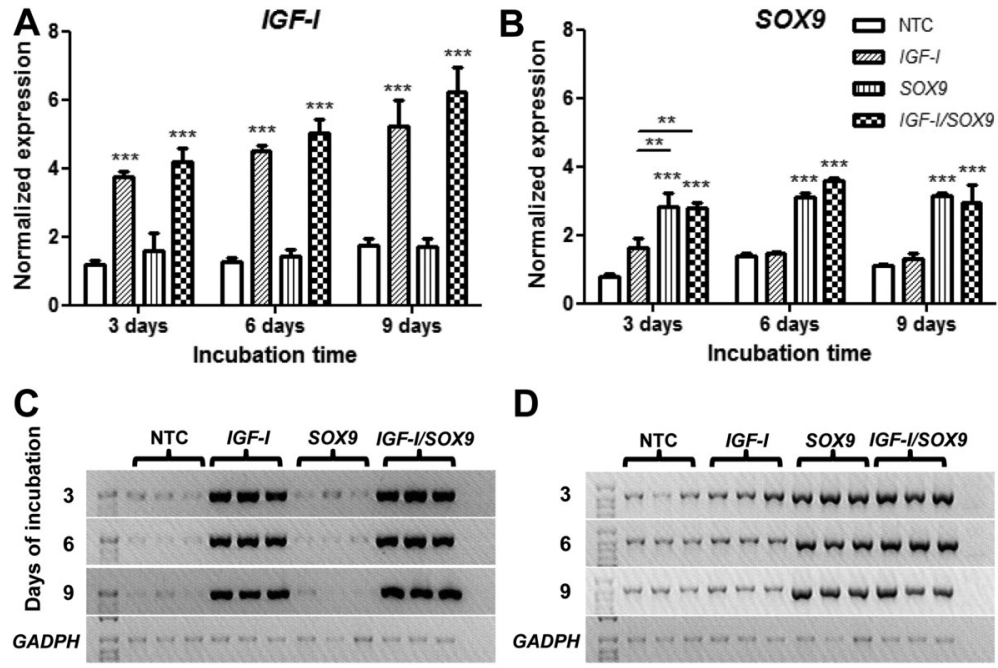

D

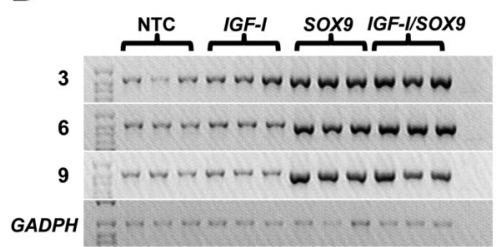

Figure 1. Chondrocyte expression of IGF-I, SOX9, and $G A P D H$. The panels show $A$, the results of IGF-I and $B$, SOX9 densitometry, and $C$ and $D$, cDNA electrophoresis bands. The columns in $A$ and $B$ indicate the amplicon densitometry from the non-transfected chondrocytes (NTC), or those transfected with pCMV-SPORT6 IGF-I (IGF-I), pCMV-SPORT6 SOX9 (SOX9), or both plasmids (IGF-I/SOX9). Data are reported as the average and standard deviation of the density values normalized to the corresponding GAPDH density of 9 assessments. ${ }^{* \star *} \mathrm{P}<0.001$, NTC vs IGF-I, SOX9 or IGF-I/SOX9 transfected chondrocytes (two-way ANOVA with Bonferroni post hoc tests). Differences in IGF-I/SOX9 and SOX9 vs IGF-I on day $\left.3{ }^{* *} P<0.01\right)$ are indicated with lines (two-way ANOVA with Bonferroni post hoc tests). 

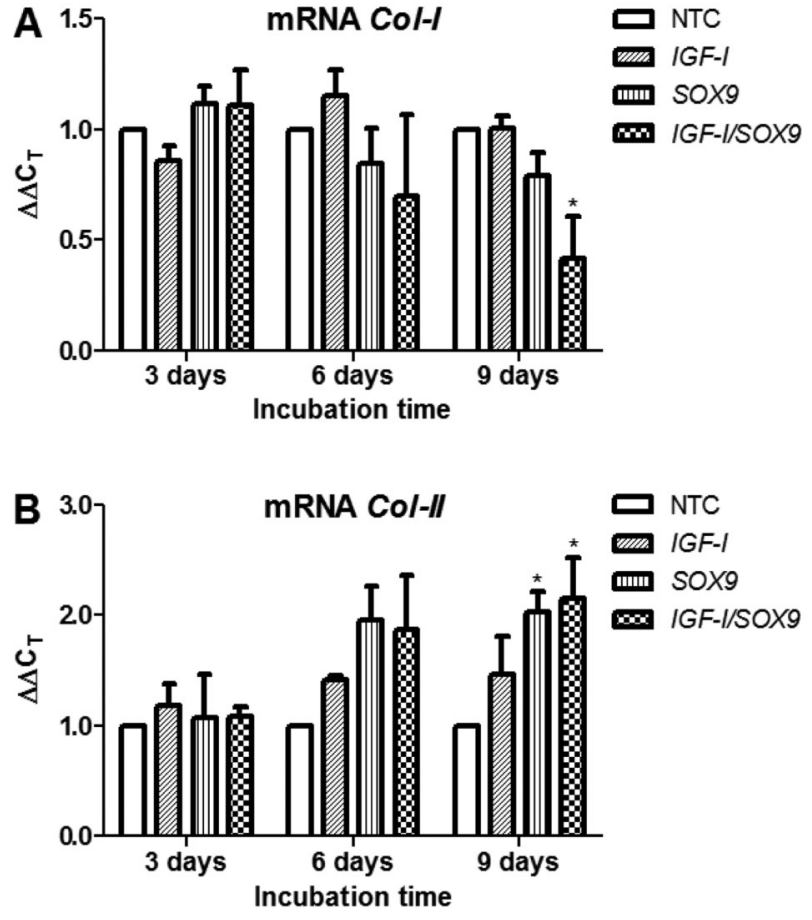

Figure 2. Relative Col-l and Col-ll gene expression by quantitative RT-PCR. Data are reported as the average and standard deviation of the 9 assessments of the expression level of the analyzed genes in non-transfected chondrocytes (NTC) or those that were transfected with pCMV-SPORT6 IGF-I (IGF-I), pCMV-SPORT6 SOX9 (SOX9), or with both plasmids (IGF-I/SOX9). The gene expressions of Col-I $(A)$ and $\mathrm{Col}-\mathrm{Il}(B)$ were normalized to the GAPDH expression levels. ${ }^{*} \mathrm{P}<0.05$ vs NTC (two-way ANOVA with Bonferroni post hoc tests).
SOX9-TC and CTC were more evident on days 6 and 9 PT. However, the cultures only showed significant differences compared with the NTC (SOX9-TC: 2.03-fold; CTC: 2.15 -fold) on day 9 PT. No significant differences were found between the NTC and the IGF-I-TC, SOX9TC, or CTC on day 6 PT (Figure 2B).

\section{Col-I and Col-II protein detection}

Immunolabeling for Col-I showed very clear positive staining in all of the chondrocyte cultures on day 3 PT. However, by day 6 PT, the Col-I-positive staining was noticeably diminished in the SOX9-TC and CTC cultures and had nearly disappeared on day 9 (Figure 3A). Conversely, all of the cells were intensely positively stained for Col-II throughout the entire observation period (Figure 4A). A morphometric analysis of these image preparations (Figures 3B and $4 \mathrm{~B}$ ) revealed no significant differences among the chondrocyte cultures on day 3 PT. On day 6 PT, the intensity of the Col-I staining was increased in all chondrocyte preparations by 1.2-2.2 times. The greatest increase in the Col-I-positive staining was observed in the NTC, and the lowest increase was noted in the SOX9-TC. The staining intensity of Col-I in SOX9-TC was significantly lower $(25 \%)$ than that of NTC. However, no significant differences were found between either IGF-I-TC or CTC and NTC. On day 9 PT, the intensities of the Col-I staining had slightly increased in the NTC and IGF-I-TC compared with those quantified in those groups on day 6 PT. In contrast, the Col-I staining intensity was significantly decreased in the SOX9-TC and CTC (2.7 times in both cases) on day 9 PT. No significant differences were found in Col-I staining between the NTC and IGF-I-TC or between the SOX9-TC and CTC (Figure 3B).
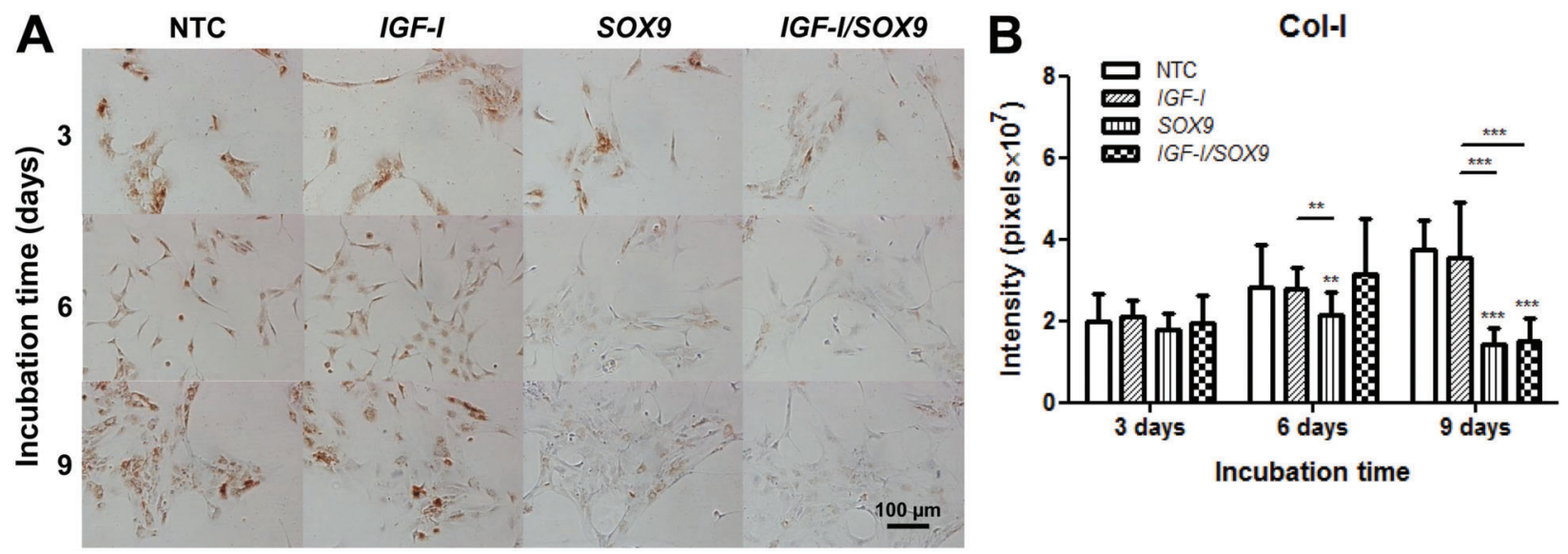

Figure 3. Immunocytochemical detection of Col-I. Specific anti-Col-I primary monoclonal antibodies $(A)$ were used to stain the non-transfected human chondrocytes (NTC) and those following transfection with pCMV-SPORT6 IGF-I (IGF-I), pCMV-SPORT6 SOX9 (SOX9), or cotransfection with both plasmids (IGF-I/SOX9). Images were taken on days 3,6, and 9 post-transfection. Once transfected, the cells were incubated at $37^{\circ} \mathrm{C}$ in a $5 \% \mathrm{CO}_{2}$ atmosphere. Quantified in situ densitometry is shown in the bar graph $(B)$. Data are reported as the average and standard deviation of the densitometry value normalized to the number of cells in the evaluated area (expressed in pixels $\times 10^{7}$ ), which was performed on all chondrocytes observed in 32 images on days 3,6 , and 9 post-transfection. ${ }^{* * *} \mathrm{P}<0.001$ and ${ }^{* *} \mathrm{P}<0.01$ vs NTC; other significant differences are indicated with lines (two-way ANOVA with Bonferroni post hoc tests). 

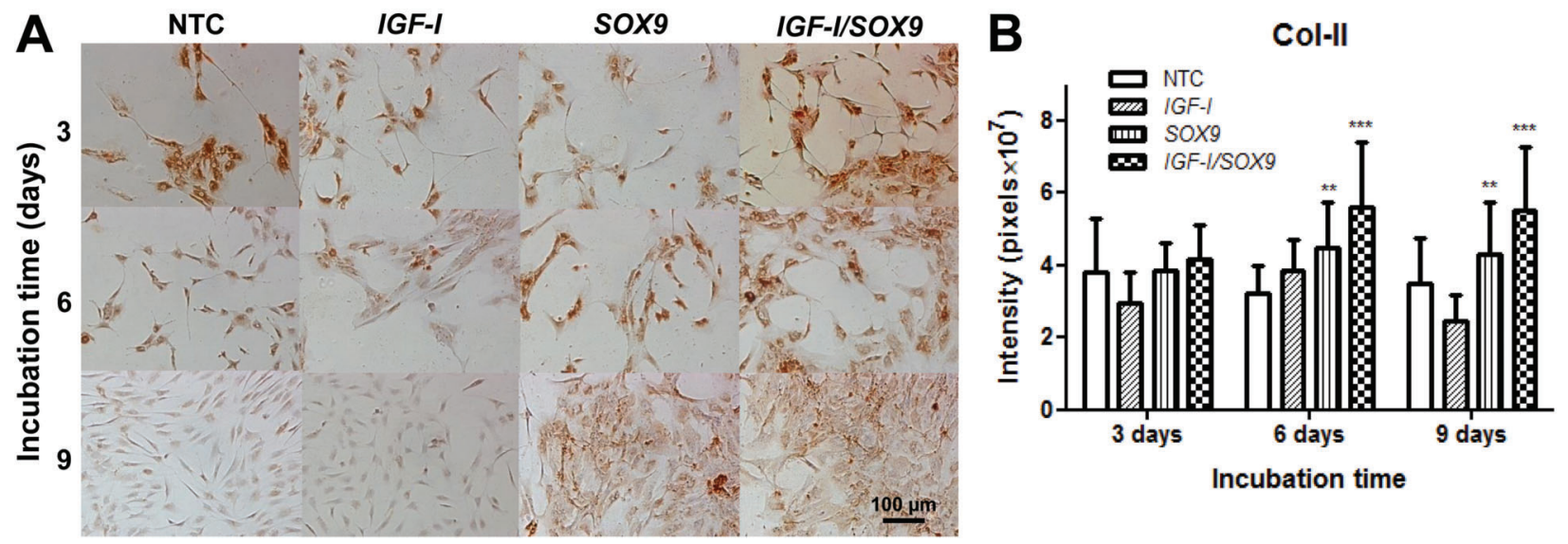

Figure 4. Immunocytochemical detection of Col-II. Specific anti-Col-Il primary monoclonal antibodies $(A)$ were used to stain the nontransfected human chondrocytes (NTC) and those transfected with plasmids pCMV-SPORT6 IGF-I (IGF-I), pCMV-SPORT6 SOX9 (SOX9), or following cotransfection with both plasmids (IGF-I/SOX9). Images were taken on days 3, 6, and 9 post-transfection. Once transfected, the cells were incubated at $37^{\circ} \mathrm{C}$ in a $5 \% \mathrm{CO}_{2}$ atmosphere. Quantified in situ densitometry is shown in the bar graph $(B)$. Data are reported as the average and standard deviation of the densitometry value normalized to the number of cells in the evaluated area (expressed in pixels $\times 10^{7}$ ), which was performed on all chondrocytes observed in 32 images on days 3,6 , and 9 post-transfection. ${ }^{* * *} \mathrm{P}<0.001$ and ${ }^{* *} \mathrm{P}<0.01$ vs NTC (two-way ANOVA with Bonferroni post hoc tests).

The morphometric analysis of Col-II (Figure 4B) showed a general increase in the Col-Il staining in the SOX9-TC and CTC, but not in the NTC. The Col-Il staining in the IGF-I-TC was lower than in that in the NTC on day 9 PT, but this difference was not statistically significant. On day $6 \mathrm{PT}$, the Col-II staining was significantly increased in the SOX9-TC (1.4 times) and CTC (1.7 times) compared with that in the NTC. The Col-II staining did not increase further on day $9 \mathrm{PT}$, but remained significantly higher than that in the NTC (SOX9-TC: 1.2 times; CTC: 1.6 times). The Col-II staining in the IGF-I-TC was lower than that in the NTC, though that difference was not statistically significant.

\section{GAG production}

As shown in Figure 5 , all of the chondrocyte cultures (NTC, IFG-I-TC, SOX9-TC and CTC) accumulated GAG in their respective culture media from days 3 to 9 PT. On day $3 \mathrm{PT}$, the GAG accumulation was minimal in the NTC culture media compared with that in the IGF-I-TC, SOX9TC, and CTC media. The accumulation of GAG in the CTC was consistently higher than those in the other three conditions, and those differences were statistically significant on days 6 and $9 \mathrm{PT}(\mathrm{P}<0.001)$. On day $9 \mathrm{PT}$, the CTC had the largest accumulation of GAG, which was significantly higher than that of the NTC (1.8 times). Significantly more GAG also accumulated in the CTC media than in the IGF-I-TC and SOX9-TC media.

\section{Discussion}

The results of this study demonstrate that the transitory cotransfection of human chondrocytes with pCMV-SPORT6
IGF-I and pCMV-SPORT6 SOX9 can be achieved with satisfactory efficiency and that cotransfection induced the simultaneous overexpression of both IGF-I and SOX9. Cotransfection of chondrocytes with these IGF-I and SOX9 vectors resulted in the overexpression of $\mathrm{Col}-\mathrm{Il}$ and the reduced expression of Col-I compared with Col-I and Col-II expression by the non-transfected chondrocytes.

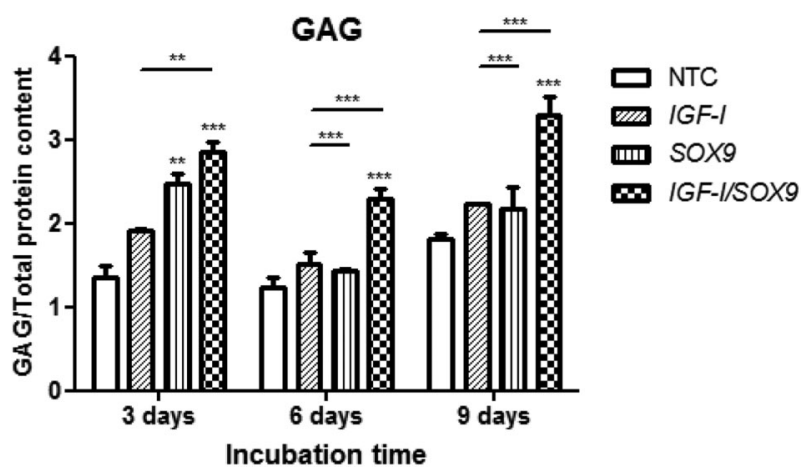

Figure 5. Glycosaminoglycans (GAG) produced by the chondrocytes. The conditioned media from each culture condition were mixed with dimethyl methylene blue dye, and the amount of GAG was quantified by spectrophotometry. The bars correspond to the non-transfected chondrocytes (NTC) and those transfected with pCMV-SPORT6 IGF-I (IGF-I), pCMV-SPORT6 SOX9 (SOX9), or both plasmids (IGF-I/SOX9). Data are reported as the mean and standard deviation of the data normalized to the total protein content based on three experiments on days 3, 6, and 9 posttransfection. ${ }^{* * *} \mathrm{P}<0.001$ and ${ }^{* *} \mathrm{P}<0.01$ vs NTC; other significant differences are indicated with lines (two-way ANOVA with Bonferroni post hoc tests). 
We selected 9 days of observation because this period was sufficient to observe the positive expression of the transgenes of interest in vivo in several expression systems $(18,19)$. SOX9 and IGF-I were overexpressed in the TC and CTC compared with their respective basal expression by the NTC. This overexpression was maintained throughout the 9-day observation period. The significantly higher number of viable cells in the IGF-I-CT and CTC than in the NTC cultures on day 9 is consistent with the well-known concept that IGF-I exerts a proliferative effect on growth plate chondrocytes (20). Interestingly, this effect was not found in the presence of cartilage matrix proteins, which is in contrast with the results here for SOX9-TC and CTC. This was likely a result of the immature state of the proliferative chondrocytes that were transfected with IGF-I. The increasing induction of Col-Il expression that was observed in the present study occurs physiologically during chondrocyte differentiation (21). The significant reduction in the expression of $\mathrm{Col}-\mathrm{I}$ on day 9 in the CTC suggests a negative transcriptional regulation of $\mathrm{Col}-\mathrm{I}$. In contrast, it is widely accepted that SOX9 positively regulates the expression of Col-II (22), while IGF-I induces Col-I/ transcription and that transcription is mediated by the SOX-trio of SOX9, SOX5, and SOX6 (23). These findings strongly suggest that during the proliferation of differentiated chondrocytes that overexpress SOX9 and IGF-I, Col-II is preferentially transcribed over Col-I. A decrease in Col-I and an increase in Col-Il proteins was clearly demonstrated in the CTC over time during incubation. This point is particularly important during articular cartilage repair because the repair tissue that fills the cartilage lesion should possess similar characteristics to hyaline cartilage. IGF-I and SOX9 each individually promoted a small accumulation of GAG in the conditioned media. Together, $I G F-I$ and SOX9 jointly stimulated a significant overproduction of GAG in the CTC compared with that in the

\section{References}

1. Roberts S, Menage J, Sandell LJ, Evans EH, Richardson JB. Immunohistochemical study of collagen types I and II and procollagen IIA in human cartilage repair tissue following autologous chondrocyte implantation. Knee 2009; 16: 398-404, doi: 10.1016/j.knee.2009.02.004.

2. Mankin HJ. The response of articular cartilage to mechanical injury. J Bone Joint Surg Am 1982; 64: 460-466.

3. Kessler MW, Ackerman G, Dines JS, Grande D. Emerging technologies and fourth generation issues in cartilage repair. Sports Med Arthrosc 2008; 16: 246-254, doi: 10.1097/ JSA.0b013e31818d56b3.

4. Madry H, Orth P, Kaul G, Zurakowski D, Menger MD, Kohn D, et al. Acceleration of articular cartilage repair by combined gene transfer of human insulin-like growth factor I and fibroblast growth factor-2 in vivo. Arch Orthop Trauma Surg 2010; 130: 1311-1322, doi: 10.1007/s00402-010-1130-3.
NTC. This strongly suggests a synergistic effect of IGF-I and SOX9. Madry et al. also stimulated Col-II and proteoglycan production (19) by cotransfecting rabbit chondrocytes with IGF-I and FGF-2, which supports the functionality of two transgenes working simultaneously in the same cells, as we described in the present study.

The transgene expression remained at a higher level than that shown by the NTC during the entire study period. This occurred despite the induction of a transient transfection using plasmids. The transitory effects of IGF-I and SOX9 on transplanted human articular chondrocytes may allow the cells to maintain their phenotype, while also providing anabolic stimuli during cell expansion before transplantation. The apparent synergistic effect of the transgenes together might be explained by the fact that these molecules are expressed together during proliferation and chondrogenic differentiation, which involves the production of proteoglycans and Col-II (24).

To our knowledge, this is the first demonstration that the simultaneous overexpression of SOX9 and IGF-I by human articular chondrocytes induced the overexpression of $\mathrm{Col}-\mathrm{Il}$ and the production of GAG, two major components of the human cartilage matrix (25). They also reduced the expression of Col-I, a major component of bone, but not of cartilage (17). These advances represent a promising alternative for cell-based therapies of human joint injuries.

\section{Acknowledgments}

The authors would like to especially thank Julio Sepúlveda-Saavedra, Ph.D., who sadly passed away during the process of this research. English-language editing of this manuscript was provided by Journal Prep. This research was supported by the Programa de Apoyo a la Investigación Científica y Tecnológica (PAICyT), Universidad Autónoma de Nuevo León. M. Simental-Mendía received a fellowship from CONACYT, Mexico.
5. Loeser RF, Pacione CA, Chubinskaya S. The combination of insulin-like growth factor 1 and osteogenic protein 1 promotes increased survival of and matrix synthesis by normal and osteoarthritic human articular chondrocytes. Arthritis Rheum 2003; 48: 2188-2196, doi: 10.1002/art. 11209.

6. Inoue A, Takahashi KA, Arai Y, Tonomura H, Sakao K, Saito $M$, et al. The therapeutic effects of basic fibroblast growth factor contained in gelatin hydrogel microspheres on experimental osteoarthritis in the rabbit knee. Arthritis Rheum 2006; 54: 264-270, doi: 10.1002/art.21561.

7. Mara CS, Duarte AS, Sartori A, Luzo AC, Saad ST, Coimbra IB. Regulation of chondrogenesis by transforming growth factor-beta 3 and insulin-like growth factor-1 from human mesenchymal umbilical cord blood cells. J Rheumatol 2010; 37: 1519-1526, doi: 10.3899/jrheum.091169. 
8. Che JH, Zhang ZR, Li GZ, Tan WH, Bai XD, Qu FJ. Application of tissue-engineered cartilage with BMP-7 gene to repair knee joint cartilage injury in rabbits. Knee Surg Sports Traumatol Arthrosc 2010; 18: 496-503, doi: 10.1007/ s00167-009-0962-2.

9. Madry H, Zurakowski D, Trippel SB. Overexpression of human insulin-like growth factor-I promotes new tissue formation in an ex vivo model of articular chondrocyte transplantation. Gene Ther 2001; 8: 1443-1449, doi: 10.1038/sj.gt.3301535.

10. Tew SR, Pothacharoen P, Katopodi T, Hardingham TE. SOX9 transduction increases chondroitin sulfate synthesis in cultured human articular chondrocytes without altering glycosyltransferase and sulfotransferase transcription. Biochem J 2008; 414: 231-236, doi: 10.1042/BJ20080262.

11. Orth P, Kaul G, Cucchiarini M, Zurakowski D, Menger MD, Kohn D, et al. Transplanted articular chondrocytes cooverexpressing IGF-I and FGF-2 stimulate cartilage repair in vivo. Knee Surg Sports Traumatol Arthrosc 2011; 19: 2119-2130, doi: 10.1007/s00167-011-1448-6.

12. Cucchiarini $M$, Terwilliger EF, Kohn D, Madry H. Remodelling of human osteoarthritic cartilage by FGF-2, alone or combined with Sox9 via rAAV gene transfer. J Cell Mol Med 2009; 13: 2476-2488, doi: 10.1111/j.1582-4934.2008.00474.x.

13. Izal I, Acosta CA, Ripalda P, Zaratiegui M, Ruiz J, Forriol F. IGF-1 gene therapy to protect articular cartilage in a rat model of joint damage. Arch Orthop Trauma Surg 2008; 128: 239-247, doi: 10.1007/s00402-007-0407-7.

14. Raper SE. Gene therapy: the good, the bad, and the ugly. Surgery 2005; 137: 487-492, doi: 10.1016/j.surg.2004.10.014.

15. Gavenis K, Schneider U, Wallich R, Mueller-Rath R, Schmidt-Rohlfing B, Andereya S. Effects of low concentrated BMP-7 administered by co-cultivation or plasmid transfection on human osteoarthritic chondrocytes. Int J Artif Organs 2010; 33: 339-347.

16. Durocher Y, Perret S, Kamen A. High-level and high-throughput recombinant protein production by transient transfection of suspension-growing human 293-EBNA1 cells. Nucleic Acids Res 2002; 30: E9, doi: 10.1093/nar/30.2.e9.
17. Clarke B. Normal bone anatomy and physiology. Clin J Am Soc Nephrol 2008; 3 (Suppl 3): S131-S139, doi: 10.2215/ CJN.04151206

18. Dinser R, Kreppel F, Zaucke F, Blank C, Paulsson M, Kochanek $S$, et al. Comparison of long-term transgene expression after non-viral and adenoviral gene transfer into primary articular chondrocytes. Histochem Cell Biol 2001; 116: 69-77.

19. Madry H, Cucchiarini M, Stein U, Remberger K, Menger MD, Kohn D, et al. Sustained transgene expression in cartilage defects in vivo after transplantation of articular chondrocytes modified by lipid-mediated gene transfer in a gel suspension delivery system. J Gene Med 2003; 5: 502-509, doi: 10.1002/jgm.368.

20. Kiepe D, Ciarmatori S, Hoeflich A, Wolf E, Tonshoff B. Insulin-like growth factor (IGF)-I stimulates cell proliferation and induces IGF binding protein (IGFBP)-3 and IGFBP-5 gene expression in cultured growth plate chondrocytes via distinct signaling pathways. Endocrinology 2005; 146: 3096-3104, doi: 10.1210/en.2005-0324.

21. Wang N, Grad S, Stoddart MJ, Niemeyer P, Sudkamp NP, Pestka J, et al. Bioreactor-induced chondrocyte maturation is dependent on cell passage and onset of loading. Cartilage 2013; 4: 165-176, doi: 10.1177/1947603512471345.

22. Akiyama $\mathrm{H}$, Lefebvre $\mathrm{V}$. Unraveling the transcriptional regulatory machinery in chondrogenesis. J Bone Miner Metab 2011; 29: 390-395, doi: 10.1007/s00774-011-0273-9.

23. Renard E, Poree B, Chadjichristos C, Kypriotou M, Maneix L, Bigot N, et al. Sox9/Sox6 and Sp1 are involved in the insulinlike growth factor-I-mediated upregulation of human type II collagen gene expression in articular chondrocytes. $\mathrm{J} \mathrm{Mol} \mathrm{Med}$ 2012; 90: 649-666, doi: 10.1007/s00109-011-0842-3.

24. Goldring MB, Tsuchimochi K, ljiri K. The control of chondrogenesis. J Cell Biochem 2006; 97: 33-44, doi: 10.1002/jcb.20652.

25. Alford JW, Cole BJ. Cartilage restoration, part 1: basic science, historical perspective, patient evaluation, and treatment options. Am J Sports Med 2005; 33: 295-306, doi: $10.1177 / 0363546504273510$. 